\title{
Frequency dependent power fluctuations: a feature of the ESR system or physical?
}

\author{
Y. Ogawa ${ }^{1}$, F. Forme ${ }^{2}$, S. C. Buchert ${ }^{1}$ \\ ${ }^{1}$ Solar-Terrestrial Environment Laboratory, Nagoya University, Furo-cho, Chikusa-ku, Nagoya, 464-8601, Japan \\ ${ }^{2}$ CETP-Universite Versailles St-Quentin en Yvelines 10-12, Av. de l'Europe, 78140 Velizy, France
}

Received: 2 February 2000 / Revised: 7 June 2000 / Accepted: 4 July 2000

\begin{abstract}
The k-dependence of the received power in high signal-to-noise ratio (SNR) conditions, occurring for naturally enhanced ion-acoustic lines (NEIALs) and for real satellites, is investigated by using the EISCAT Svalbard Radar (ESR), where the data are recorded in eight separate channels using different frequencies. For the real satellites we find large variations of the relative powers from event to event, which is probably due to a different number of pulses catching the satellite over the integration period. However, the large power difference remains unexpected in one case. Over short time scale $(\leq 10$ s) the relative power difference seems to be highly stable. For most NEIAL events the differences between channels are within noise level. In a few cases variations of the relative power well above both the estimated and expected 1-sigma level occur over a signal preintegrated profile. We thus suggest that the frequency dependence of the power in NEIAL events has its origin in the scattering medium itself as the most plausible explanation.
\end{abstract}

Key words: Ionosphere (auroral ionosphere; plasma waves and instabilities; instruments and techniques)

\section{Introduction}

Incoherent scatter (IS) radar signals sometimes show spectra which cannot be explained by the IS theory. These spectra have enhanced power at the ion acoustic frequency, therefore they are called naturally enhanced ion-acoustic lines (NEIALs). Rietveld et al. (1991) presented a systematic study of NEIALs received by the EISCAT UHF system. Several models to explain NEIALs have been proposed such as the current driven

Correspondence to: Y. Ogawa

e-mail: yogawa@stelab.nagoya-u.ac.jp instability (Foster et al., 1988) and the ion-ion twostream instability (Wahlund et al., 1992). To decide whether and how the cross section of NEIALs depends on the wave vector $\mathrm{k}$ may enable us to distinguish between such instabilities. Cabrit et al. (1996) first discussed the k-dependence of the NEIAL cross section using the EISCAT VHF and UHF systems simultaneously. Buchert et al. (1999) made use of data recorded separately from different receiver channels of the ESR, which are spaced over frequency interval of $2 \mathrm{MHz}$. They suggested that the power of NEIALs varies strongly with wavelength. Such a strong dependence of $\mathrm{k}$ may be explainable by a model involving the parametric decay of beam driven Langmuir wave (Forme, 1993). In this study we use a larger data set of NEIALs events and also compare their power characteristics with those of real satellite echos.

\section{Motivation}

Figure 1 shows power profiles of incoherent scatter signals from the GUP0 program. In the left panels the thick line indicates data from channel 1 using the lowest frequency of the ESR, and the thin line indicates data from channel 6 using the highest frequency of the ESR. In the case of normal incoherent signals (upper left panel), there seems to be no significant difference between the power from both channels. The right panels show the normalized power difference between the two channels. This normalized difference increases with range, because the SNR decreases with range thus the statistical fluctuations increase. For low SNR statistical power differences between two channels are relatively large and randomly fluctuating. This is expected because the GUP0 receiver settings produce only small correlations between subsequent samples for a noise-dominated input. For high SNR the power differences are small and seem somewhat correlated which is also expected for an IS signal with a poor range resolution of $54 \mathrm{~km}$.

On the other hand, in the lower left panel NEIALs enhance the power in range between 400 and $800 \mathrm{~km}$. In 
(a) 072410 UT

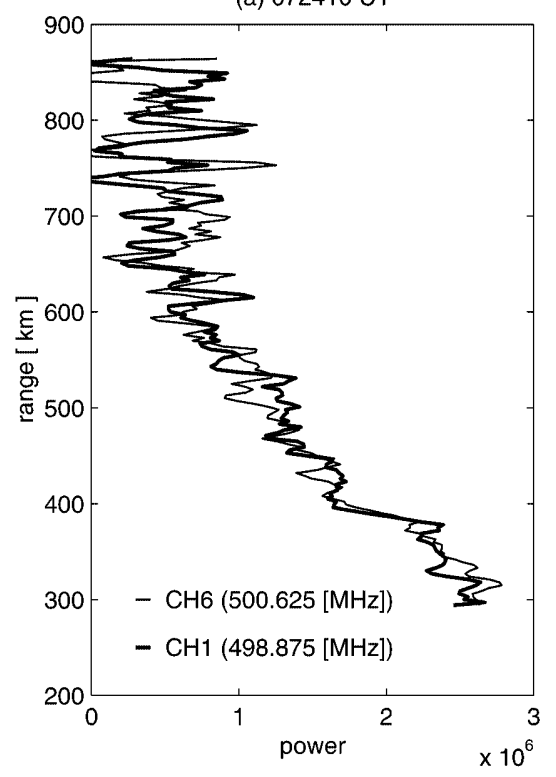

(c) 072320 UT

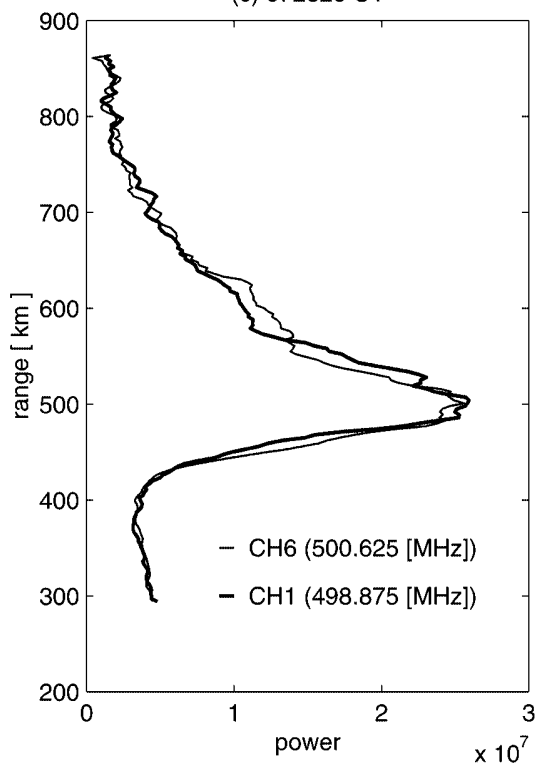

(b) 072410 UT

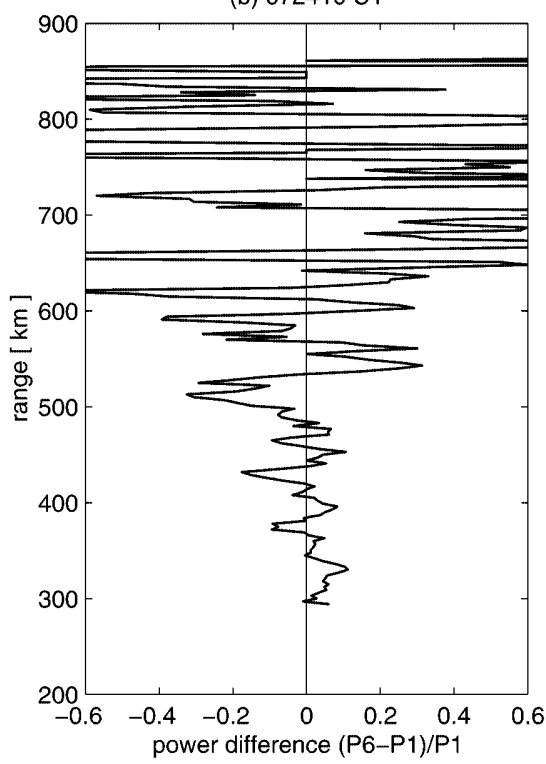

(d) 072320 UT

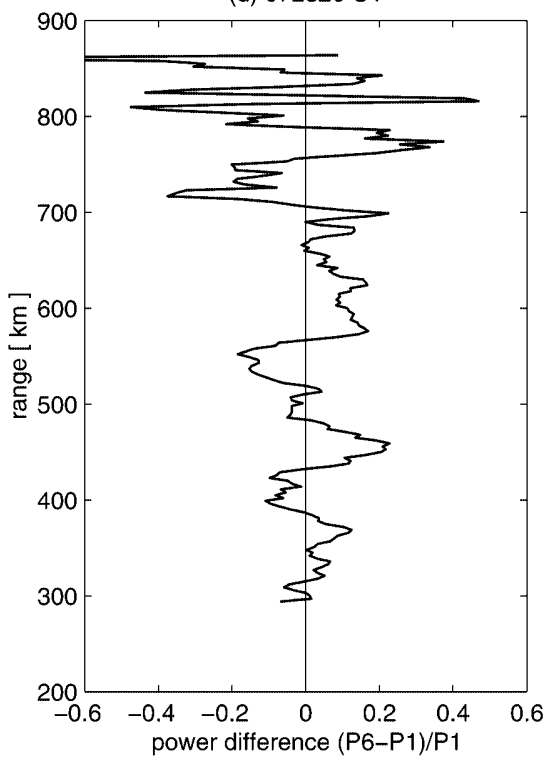

Fig. 1a, b. Power profiles from two different receiver channels. The power of channel 6 has been corrected for the relative gain difference (see text in Sect. 3). (a) Normal IS signals and (b) naturally enhanced ionacoustic lines (NEIALs). The right panels show normalized power differences the case of the NEIALs, the received power from both channels is relatively high, causing a high SNR. Note that the power scale (x axis) in the lower panel is 10 times larger than in the upper panel. Normal incoherent signals are seen around the $F 2$ peak and the normalized power difference from the two channels (right panel) is within the statistical fluctuations. However, around the power peak $(500 \mathrm{~km})$, the power from both channels seem to differ more than we expect, taking into account that the SNR is very high compared to the upper panel. For such a high SNR we expect rather small relative power differences, smaller than in the same profile at around $300 \mathrm{~km}$. At the largest ranges around $850 \mathrm{~km}$ there is still significant power in the noise-subtracted profile with NEIALs (lower panel in Fig. 1). We have observed that this is a typical feature of profiles with NEIALs and it is absent in profiles with only IS signals.
The NEIALs seem to produce a distinct power peak but also to extend over a wider range consistent with earlier studies (Rietveld et al., 1991). They are rather a soft radar target filling the volume illuminated by the radar pulses as opposed to real satellites which are hard targets. If the power differences of a soft target were fluctuating uncorrelated along the profile, the differences should tend to cancel when a long pulses $(54 \mathrm{~km}$ in this experiment) are used, although some correlation between samples arises from the uneven power level along the illuminated volume, similar the situation in the upper panel for IS signals. But the relatively smoothly varying, large power difference as seen in Fig. 1 between about 400 and $700 \mathrm{~km}$ cause us to suspect, that NEIALs not only exhibit larger power differences than expected, but that the differences seem to be systematic along a profile. 
Table 1. List of channels of GUP0

\begin{tabular}{llll}
\hline Channel & $\begin{array}{l}\text { Frequency } \\
(\mathrm{MHz})\end{array}$ & $\begin{array}{l}\text { Pulse length } \\
(\mu \mathrm{s})\end{array}$ & $\begin{array}{l}\text { Range } \\
(\mathrm{km})\end{array}$ \\
\hline 1 & 498.875 & 360 & $294-921$ \\
2 & 500.125 & 360 & $237-864$ \\
3 & 499.375 & 360 & $180-807$ \\
4 & 500.375 & 150 & $123-750$ \\
5 & 499.625 & 360 & $294-921$ \\
6 & 500.625 & 360 & $237-864$ \\
7 & 499.875 & 360 & $180-807$ \\
8 & 500.875 & 150 & $123-750$ \\
\hline
\end{tabular}

In the following we investigate whether these power difference are related to the occurrence of high SNR or perhaps an inherent feature of NEIALs. Furthermore, we assess how often these k-dependence occur. In this study, the k-dependence of NEIALs is investigated using a small data base of NEIAL and satellite events in ESR data.

\section{Method}

The relatively low peak but high average power $(25 \%$ duty cycle) of the ESR transmitter can be utilized
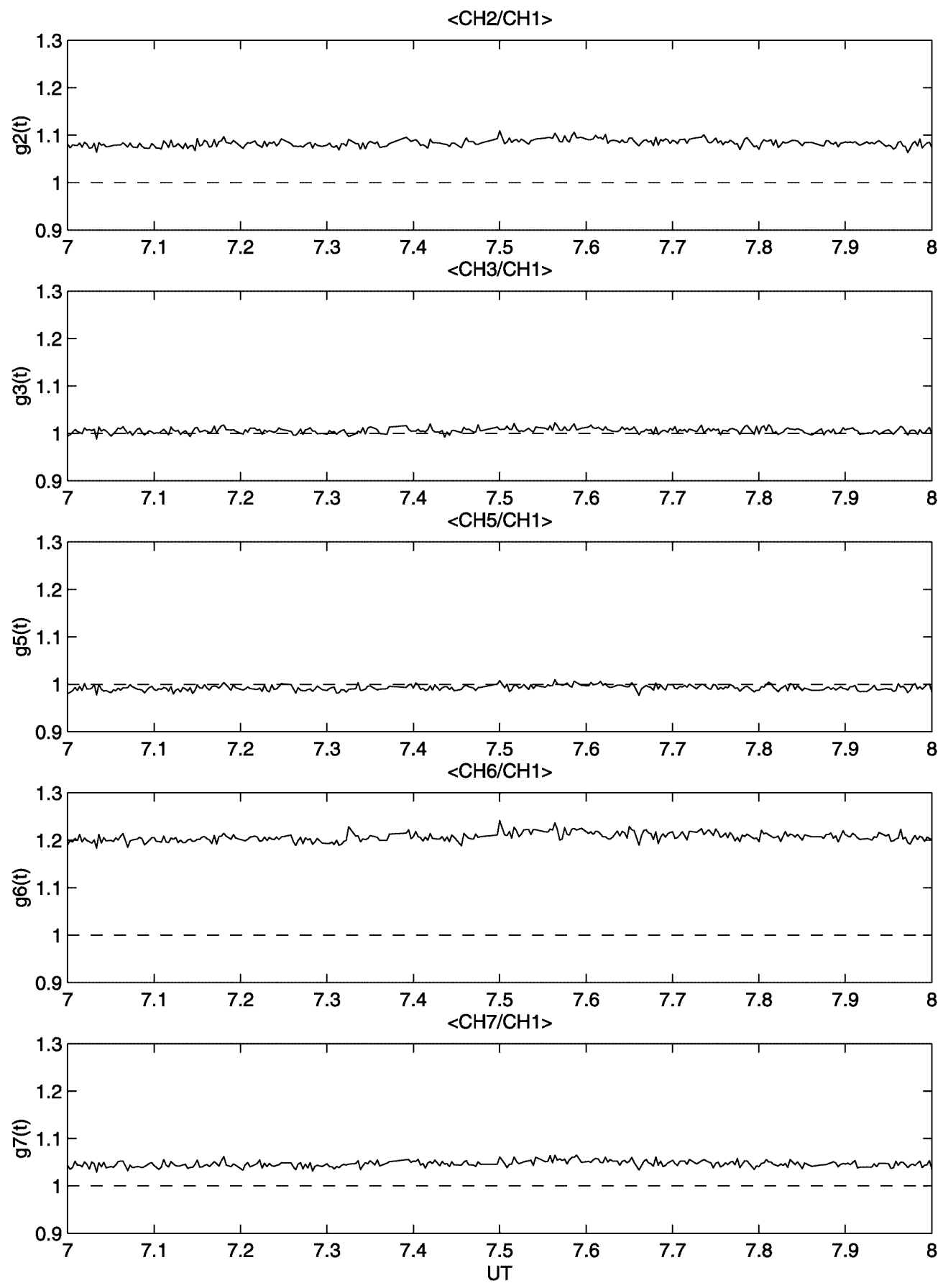

Fig. 2. Estimated relative gains between channels over time between 7 and 8 UT 
efficiently only through systematic use of multi-frequency modulations (Wannberg et al., 1997). Among the basic radar codes that were constructed for the ESR, GUP0 code is the most suitable one to compare the power difference between channels. The GUP0 is a multi-frequency long-pulse code, and mainly intended for $F$ region and topside observations.

Table 1 shows a summary of the GUP0 code. The maximum frequency difference between eight channels around $500 \mathrm{MHz}$ is $2 \mathrm{MHz}$. In this study, we used channels $1,2,3,5,6$ and 7 , because they receive pulses of equal length $(360 \mu \mathrm{s})$ with a range resolution of $54 \mathrm{~km}$. The other two channels use a pulse length of $150 \mu \mathrm{s}$. For our investigation, we have used data between 7 and 8 UT on July 6, 1998, because in this period many samples of NEIALs were seen. The antenna was field-aligned in this experiment. In a sophisticated radar like the ESR the signals pass through many stages. It is difficult to calculate the expected relative gain from two different channels. Therefore we attempt to use the data themselves to calibrate and assume the following relation between channels:

Power $_{n}=g_{n}(t) \cdot$ Power $_{1}$,

$g_{n}(t)=\left\langle\frac{\text { background }_{n}(t)}{\text { background }_{1}(t)}\right\rangle_{r}$,

where $n$ indicates the channel number, and $g_{n}(t)$ is an estimate of the relative gain during one preintegration period which may depend on the time $t$. The subscript $r$ indicates that averaging is made over a preintegrated power profile using the SNR to weight the data.

The five panels in Fig. 2 show the relative $g_{n}(t)$ from 7 to 8 UT. $g_{n}$ seems to depend only weakly on time. Histograms in Fig. 3 show the distributions of the relative gains. All distributions are consistent with the assumption that the relative gains have simply statistical fluctuations. We use the average of each distribution to compare the measured power at different channels/ frequencies.

\section{Results}

In Fig. 4, five scatter plots show relative power differences over SNR. Thin points are from normal IS signals, and thick ones at the high SNR are from ranges with power enhancements from NEIALs. NEIALs were always seen on all channels. Assuming a Gaussian distribution of both the noise and the scatter signal, data should scatter approximately according to the relation

$\frac{\Delta \text { Power }^{\text {Power }_{1}}}{=} \frac{1}{\sqrt{N(I P P)}} \cdot\left(1+\frac{1}{S N R}\right)$.

$N(I P P)$ is the number of samples. One effective interpulse period (IPP) lasts $13440 \mu \mathrm{s}$. The autocorrelated data are integrated over 744 IPPs or $10 \mathrm{~s}$. We have also estimated the standard deviation of the relative differ- (a) $<\mathrm{CH} 2 / \mathrm{CH} 1>$

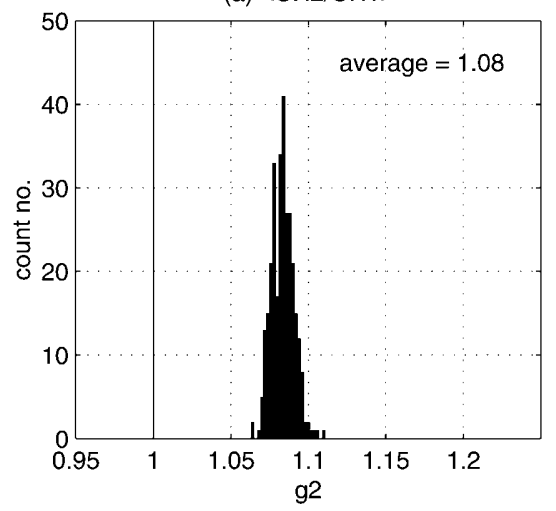

(d) $<\mathrm{CH} 6 / \mathrm{CH} 1>$

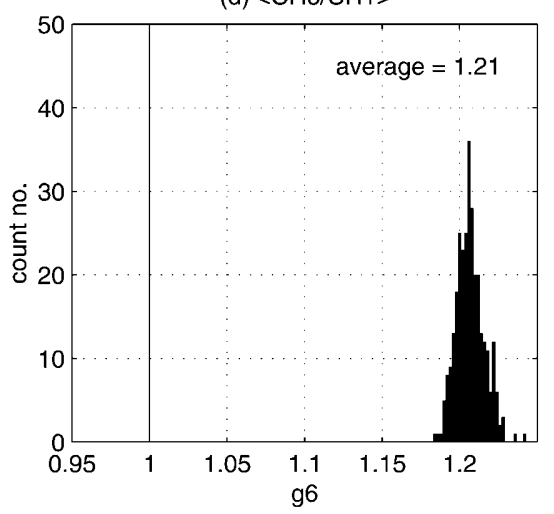

(b) $\langle\mathrm{CH} 3 / \mathrm{CH} 1\rangle$

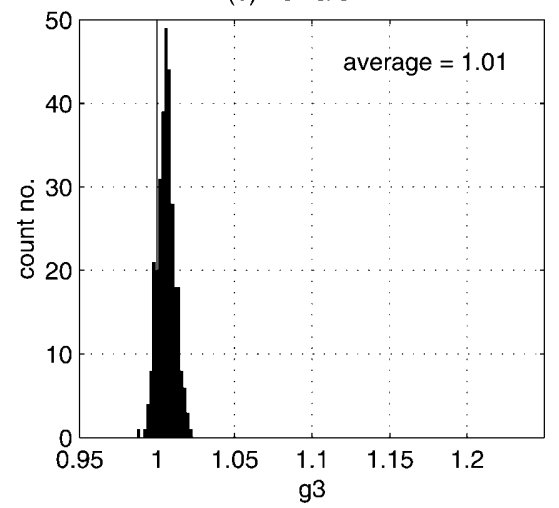

(e) $<\mathrm{CH} 7 / \mathrm{CH} 1>$

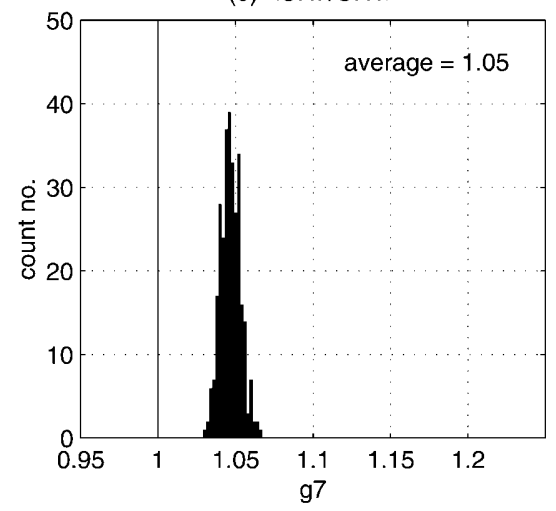

(c) $<\mathrm{CH} 5 / \mathrm{CH} 1>$

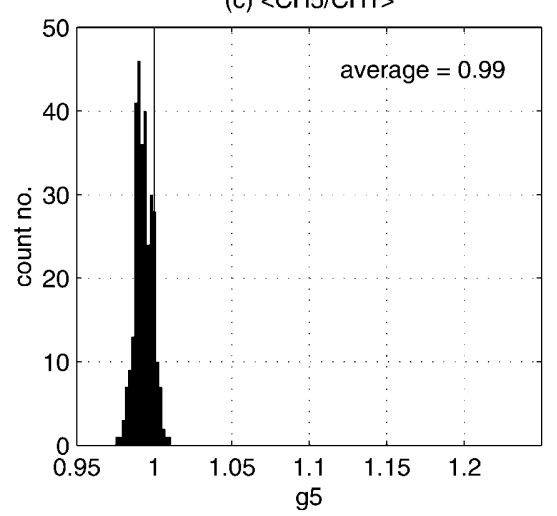

Fig. 3a-e. Distributions of the relative gains, over the time period shown in Fig. 2 
(a) $(\mathrm{CH} 2-\mathrm{CH} 1) / \mathrm{CH} 1$

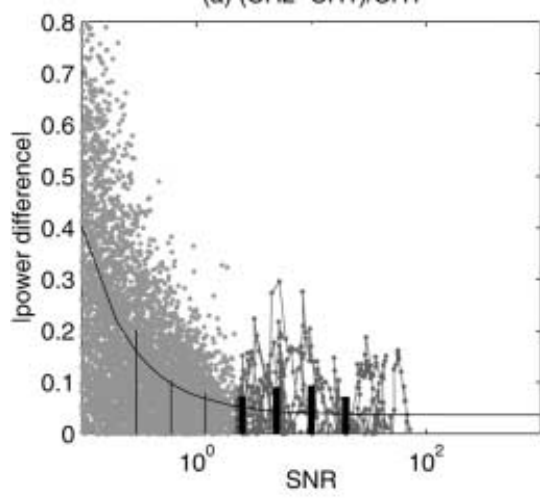

(d) $(\mathrm{CH} 6-\mathrm{CH} 1) / \mathrm{CH} 1$

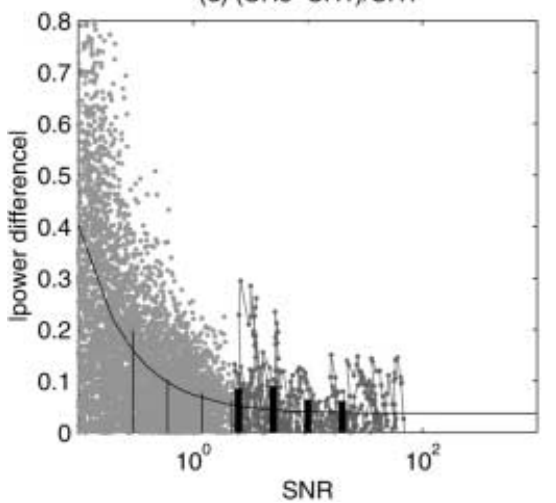

(b) $(\mathrm{CH} 3-\mathrm{CH} 1) / \mathrm{CH} 1$

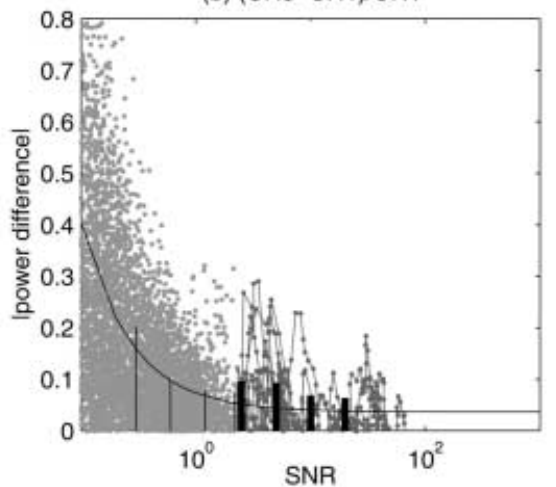

(e) $(\mathrm{CH} 7-\mathrm{CH} 1) / \mathrm{CH} 1$

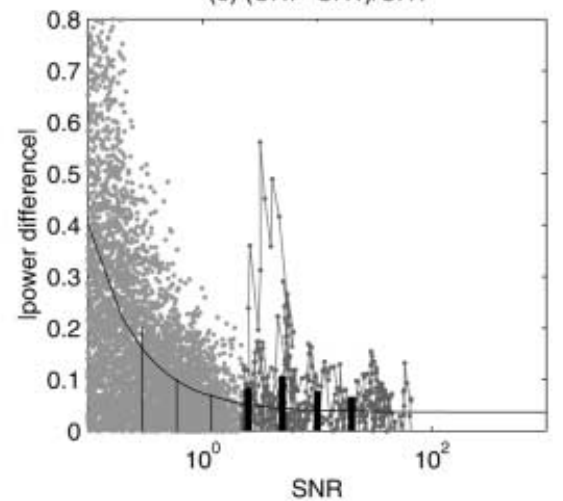

(c) $(\mathrm{CH} 5-\mathrm{CH} 1) / \mathrm{CH} 1$

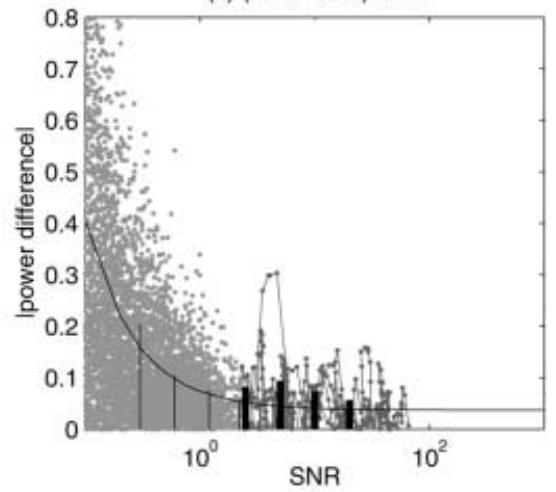

Fig. 4. Relative power differences between channels over SNR. Vertical bars indicate the 1-sigma levels that are calculated from the distribution of data points falling within corresponding SNR bins. The boundaries of the SNR bins are 0.2, 0.5, 1, 1.5, 3, 7, 20 and 100 .
Gray dots are from normal IS signals, and thin vertical bars indicate the respective 1-sigma levels. Black dots (connected by lines) are from NEIAL data, and the thick vertical bars indicate their respective 1-sigma levels. The curve represents Eq. (3) ences in 8 SNR bins and found empirically that relation (3) represents reasonably well the actual standard deviation. Most data points, in particular at SNR $<3$, scatter not more than expected from normal distributions with standard deviation given by the curve in Fig. 4. Enhanced (thick) data points connected by a line originate from the same profile (preintegration period). It can be seen that the data points with large power differences come from the same profiles. In other words, where there is a power difference larger than statistically expected, it also tends to vary systematically with a altitude over one postintegration period. If all the enhanced power of NEIALs were produced in a narrow layer, similarly as for a hard target, the long pulse length combined with the receiver filter would lead to horizontally rather than vertically connected data points as will be shown in the next section for real satellites. It should be noted that our NEIALs last sometimes $30 \mathrm{~s}$ and longer, consistent with the EISCAT UHF observations (Rietveld et al., 1991). NEIALs typically seem to come and go over seconds or tens of seconds. If they were made of conglomerations of very short-lived $(\leq 1 \mathrm{~s})$ bursts, each one producing a much higher SNR than the observed, average SNR, a random frequency dependency of the cross section should tend to cancel when integrating over $10 \mathrm{~s}$.

In order to obtain a statistically meaningful quantity for the difference between NEIAL and normal IS data, we have mapped all data points in Fig. 4 to a reference SNR of 1 using Eq. (3). Thus two distributions of power differences are created, one for normal IS and one for NEIALs. They are shown in Fig. 5. These distributions are not composed of completely independent points because the long pulse length causes correlations along a profile as discussed qualitatively in Sect. 2. We have assumed that only every 18 th point is independent, which is pessimistic, since data points are obviously not correlated over a complete pulse length. Using the test described by Press et al. (1997) there is a $10 \%$ or lower probability that both distributions in Fig. 5 are the same or that NEIALs statistically do not have large power differences than IS signals. Next we investigate the relative power in real satellites which also cause high, often even higher SNR than NEIALs.

\section{Comparison with echos from satellites}

Echos from satellites can easily be identified, because (1) their spectrum has very sharp lines unlike the ion lines, (2) the satellite return is seen over a range of $54 \mathrm{~km}$ which is the length of the pulse.

We found two real satellites in the data period which was also used for the NEIAL study. Figure 6 shows relative power differences between channels for real satellites in the same format as Fig. 4. The power 

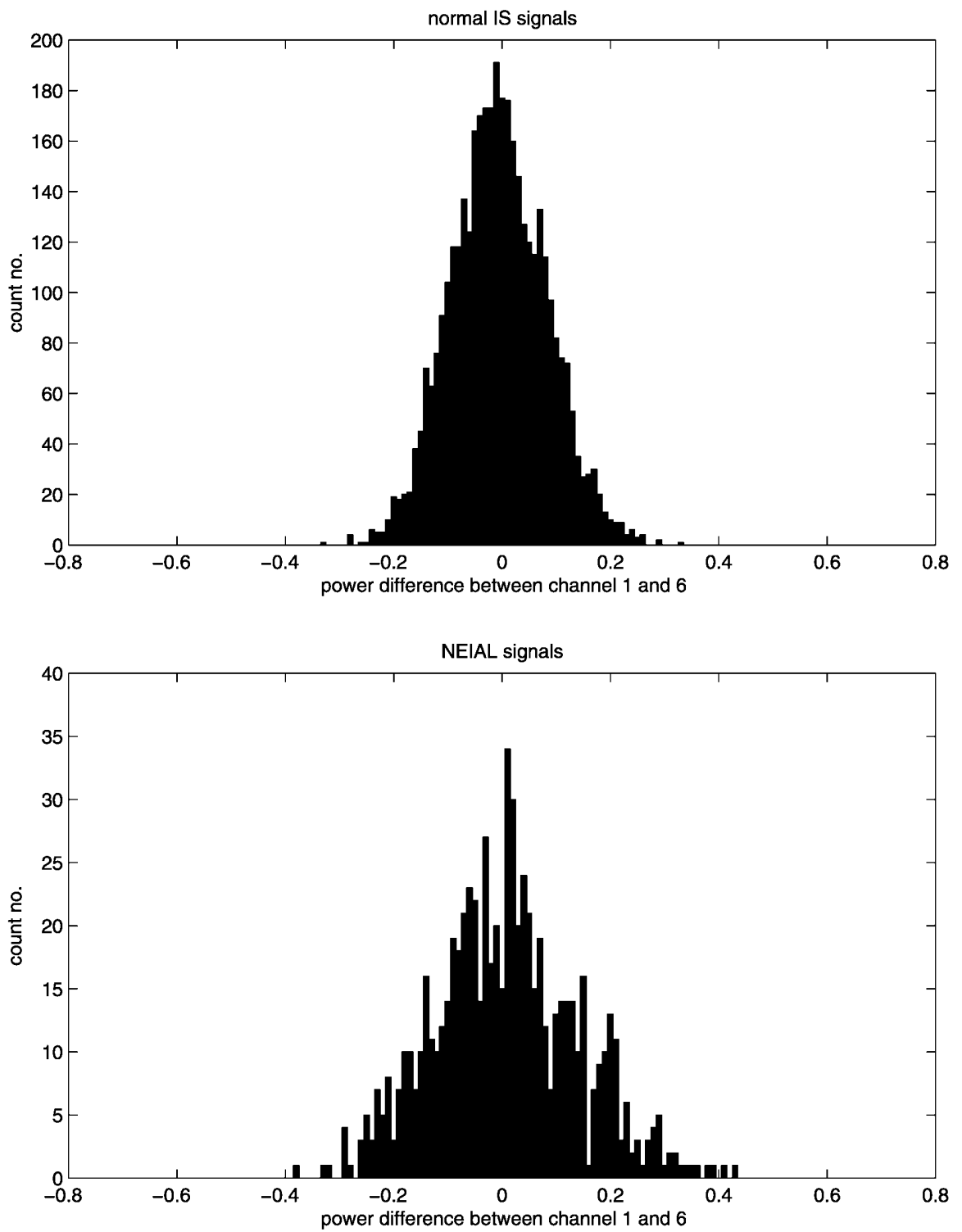

Fig. 5. Distributions of the power differences between channel 1 and 6 in normal IS signals (upper panel) and in NEIAL signals (lower panel). Each power difference is mapped by using Eq. (3) to a SNR = 1 differences follow approximately a horizontal line for each profile containing a satellite echo which is mainly due to the small correlations induced by the digital ESR receiver filters. Most satellite echos are in the right tips of the approximately horizontal lines. This means that the power difference is constant over an integration period, even if the SNR gets very high. This is obviously different from the behavior for NEIALs. We thus conclude that the relative gains in the receiver do not vary systematically over a pulse cycle within a few interpulse periods, but is rather constant over such short time scales $(13440 \mu \mathrm{s})$. The relative power in real satellites, although constant over a preintegrated profile, varies from event to event by a large factor, much more than expected from Fig. 2. In GUP0 the pulses from channels 1,2, and 3 are transmitted in one IPP, and from channels 5, 6, and 7 in the next IPP. A satellite may enter or leave the radar beam rapidly within $100 \mathrm{~ms}$ or so between pulses from defferent channels, explaining large power difference between channel 1 and 5,6, and 7 , respectively. This, however, leaves the large difference between the two events comparing channels 1 and 2 with in time adjacent transmitter pulses in the upper left panel of Fig. 6 unexpected and unexplained.

\section{Discussion and conclusion}

We have studied the relative powers between channels using different frequencies in high SNR situations. In case of NEIALs possible power differences are usually within the noise level, but we found two profiles within $1 \mathrm{~h}$ of data where the power radiated by NEIALs varies systematically with wavelength. This feature of NEIALs does not seem to be caused by the ESR system. More samples are needed for a future study. Furthermore, power differences along one profile with NEIALs are obviously different from that of echoes from satellites at similarly high SNR. Therefore the NEIAL cross section itself seems to vary strongly with frequency. If the NEIAL power is highly frequency dependent, why are the differences usually in the noise level? It may be due 
(a) $(\mathrm{CH} 2-\mathrm{CH} 1) / \mathrm{CH} 1$

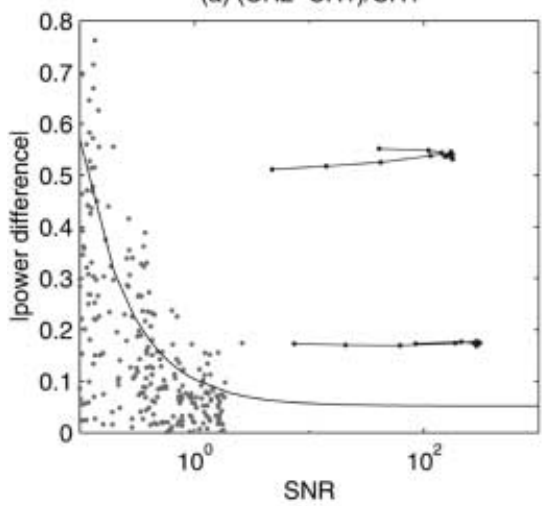

(d) $(\mathrm{CH} 6-\mathrm{CH} 1) / \mathrm{CH} 1$

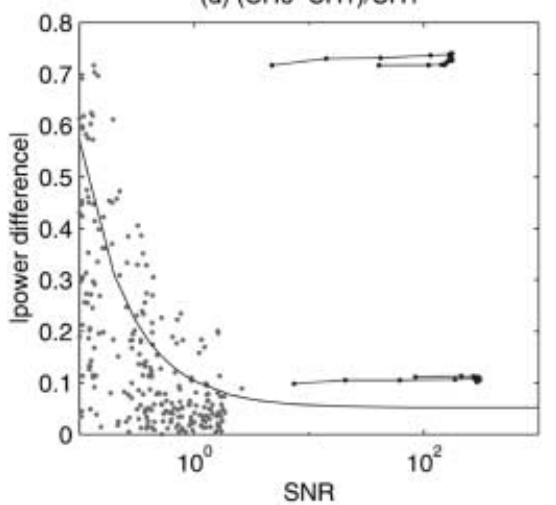

(b) $(\mathrm{CH} 3-\mathrm{CH} 1) / \mathrm{CH} 1$

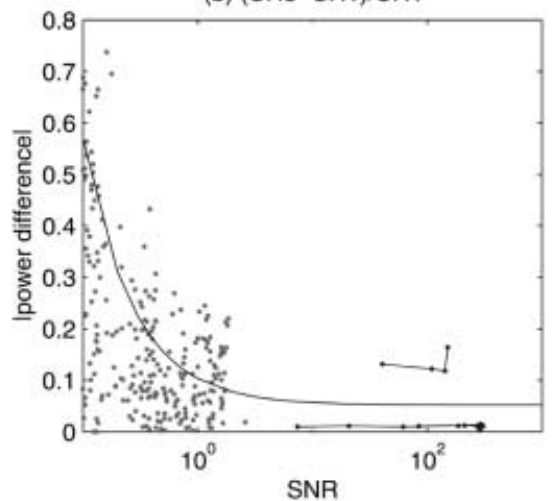

(e) $(\mathrm{CH} 7-\mathrm{CH} 1) / \mathrm{CH} 1$

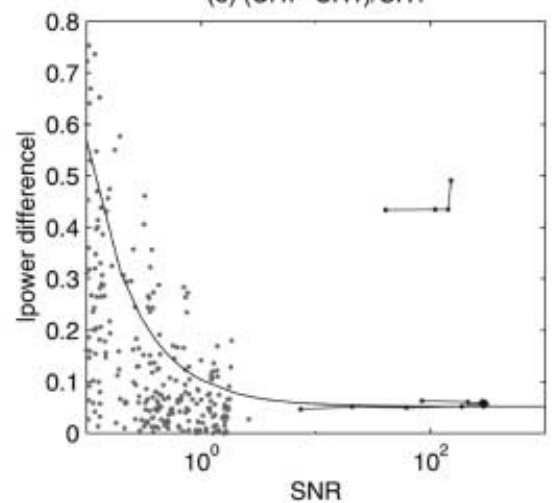

(c) $(\mathrm{CH} 5-\mathrm{CH} 1) / \mathrm{CH} 1$

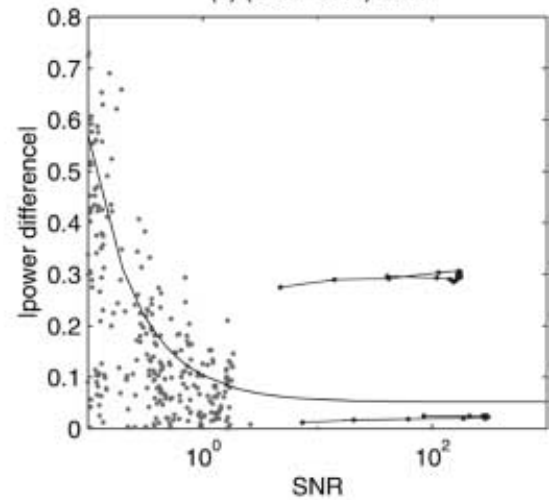

Fig. 6a-e. Relative power differences for real satellites in the same format as Fig. 4. Lines at the high SNR are from ranges with power enhancements from real satellites

to the very small frequency spacing. The relative frequency difference is about $0.3 \%$. We plan to use a wider frequency spacing between channels. The GUP3 code, adopted recently as a common program of the ESR, uses only two long pulses, and its frequency difference is about $0.5 \mathrm{MHz}$. Although there are a lot of data using the GUP3 code, they are not useful for this kind of study.

An application for a frequency assignment at $500 \pm 2 \mathrm{MHz}$ was submitted to the Norwegian frequency planning authority (Wannberg et al., 1997). Therefore the maximum frequency difference we can use is 4 $\mathrm{MHz}$. This difference is twice as large as that of the GUP0 code. A special experiment with wider frequency spacing than that of GUP0 will be completed to investigate the frequency dependency of NEIAL power in more detail.

Acknowledgements. We are indebted to the director and staff of EISCAT for operating the facility and supplying the data. EISCAT is an international association supported by Finland (SA), France (CNRS), the Federal Republic of Germany (MPG), Japan (NIPR), Norway (NFR), Sweden (NFR), and the United Kingdom (PPARC). This study was supported in part by grants 09044074 , 08304030, 08454135, Ministry of Education, Science, Sports and Culture, Japan.

Topical Editor M. Lester thanks C.J. Heinselman and another referee for their help in evaluating this paper.

\section{References}

Buchert, S. C., A. P. van Eyken, T. Ogawa, and S. Watanabe, Naturally enhanced ion-acoustic lines seen with the EISCAT Svalbard Radar, Adv. Space Res. (in press), 1999.

Cabrit, B., H. Opgenoorth, and W. Kofman, Comparison between EISCAT UHF and VHF backscattering cross section, J. Geophys. Res., 101, 2369-2376, 1996.

Foster, J. C., C. Del Pozo, K. Groves, and J.-P. St-Maurice, Radar observations of the onset of current driven instabilities in the topside ionosphere, Geophys. Res. Lett., 15, 160-163, 1988.

Forme, F. R. E., A new interpretation on the origin of enhanced ion acoustic fluctuations in the upper ionosphere, Geophys. Res. Lett., 20, 2347-2350, 1993.

Press, W. H., S. A. Teukolsky, W. T. Vetterling, and B. P. Flannery, Numerical Recipes in C, 2nd edn, Cambridge University Press, Cambridge, UK, 1992.

Rietveld, M. T., P. N. Collis, and J.-P. St.-Maurice, Naturally enhanced ion acoustic waves in the auroral ionosphere observed with the EISCAT $933 \mathrm{MHz}$ radar, J. Geophys. Res., 96, 19 29119 305, 1991.

Wahlund, J.-E., F. R. E. Forme, H. J. Opgenoorth, M. A. L. Persson, E. V. Mishin, and A. S. Volokitin, Scattering of electromagnetic waves from a plasma: enhanced ion acoustic fractuations due to ion-ion two-Stream Instabilities, Geophys. Res. Lett., 19, 1919-1922, 1992.

Wannberg, G., I. Wolf, L.-G. Vanhainen, K. Koskenniemi, J. Rottger, M. Postila, J. Markkanen, R. Jacobsen, A. Stenberg, R. Larsen, S. Eliassen, S. Heck, and A. Huuskonen, The EISCAT Svalbard radar: a case study in modern incoherent scatter radar system design, Radio Sci., 32, 2283-2307, 1997. 